\title{
The impact of Better Conversations with Aphasia on current practice by UK speech and language therapists
}

Suzanne Beeke ${ }^{1}$, Nicola Sirman ${ }^{2}$, Firle Beckley ${ }^{1}$, Jane Maxim ${ }^{1}$, Susan Edwards ${ }^{1,3}$ \& Wendy Best ${ }^{1}$

1. Division of Psychology and Language Sciences, University College London, UK

2. Solent NHS Trust, Southampton, UK

3. School of Psychology and Clinical Language Sciences, University of Reading, Reading, UK

Corresponding author s.beeke@ucl.ac.uk

Background: Better Conversations with Aphasia (BCA; Beeke et al 2013) is a freely available conversation therapy programme underpinned by conversation analytic theory that currently has more than 5,400 registered users worldwide, approximately $60 \%$ of whom are in the UK. It engages both a person with aphasia (PWA) and their conversation partner (CP) in video feedback exercises to reinforce good conversation strategies, and to identify barriers to conversation. They then jointly set goals to practise up to three facilitatory behaviours in coached conversation tasks with the speech and language therapist (SLT). There is evidence of the effectiveness of BCA, namely a significant reduction in barriers to conversation at the group level, and for some PWAs and their CPs, a significant increase in facilitatory conversation behaviours (Best et al 2016). The online resource provides SLTs with access to the BCA intervention programme and training materials including videos showing BCA therapy being delivered, guidance on goal-setting and outcome measurement, and advice from experienced SLTs. It. However, it is not clear how current BCA practice compares with the published research evidence, both for BCA and for CP training in general (Simmons-Mackie et al 2016). For example, Sirman et al (2017) reported a significant evidence-to-practice gap for conversation therapy practice by UK SLTs. It is also pertinent and timely to explore the factors that influence the implementation of BCA in UK practice.

Aims: To explore current BCA practice by UK SLTs. To investigate facilitators and barriers to the implementation of BCA by SLTs in practice.

Methods \& Procedures: An online survey is underway. The survey inclusion criterion is qualified SLTs practising in the UK who are registered users of the BCA resource. The aim is to target $20 \%$ of UK users, aiming for 600 respondents. A mixture of question types including multiple choice, rating of statements and open-ended focus on (1) participant demographics; 2) general BCA resource use; 3) BCA delivery, including client groups, settings, what outcomes are measured, techniques (video feedback, practice), materials (handouts, personal videos, resource videos), acceptability to client/CP; 4) knowledge, understanding and confidence of the SLT prior to and after use of the BCA resource; 5) facilitators and barriers to implementation, 6) additional comments. Data analysis will involve descriptive statistics (demographics, frequency of use, client groups and settings, modules accessed etc.) and thematic content analysis of respondents' views on resource utility and suggestions for improvement.

The project is covered by BCA programme approval from the UCL Language and Cognition Departmental Ethics Committee.

Outcomes \& Results: The findings for current practice will be compared with research evidence for the BCA approach and for CP training, in terms of targeted clients and CPs, candidacy, dose, and techniques (video feedback, practice) following aspects of the taxonomy developed by SimmonsMackie et al (2014), to characterize and contrast key elements of conversation therapy for aphasia. 
(Simmons-Mackie et al 2014). The top three barriers and facilitators to implementation will be reported.

Conclusions: This project addresses directly the key issue of a known gap between the evidence base for BCA, and CP training in general, and SLT clinical practice in the UK. Key findings will be further explored via focus groups with SLTs who complete the survey. The results will help to plan a further international survey, taking into account the complexities of implementation across other healthcare systems.

References:

Beeke S, Sirman N, Beckley F, Maxim J, Edwards S, Swinburn K, Best W. (2013). Better Conversations with Aphasia: an e-learning resource. Available at: https://extend.ucl.ac.uk/

Best W, Maxim J, Heilemann C, Beckley F, Johnson F, Edwards SI, Howard D and Beeke S (2016) Conversation Therapy with People with Aphasia and Conversation Partners using Video Feedback: A Group and Case Series Investigation of Changes in Interaction. Frontiers in Human Neuroscience 10:562. doi:10.3389/fnhum.2016.00562

Simmons-Mackie, N., Savage, M.C. and Worrall, L., (2014) Conversation therapy for aphasia: a qualitative review of the literature. International Journal of Language \& Communication Disorders, 49(5), 511-26.

Simmons-Mackie, N., Raymer, A., and Cherney, L. R. (2016). Communication partner training in aphasia: An updated systematic review. Archives of Physical Medicine and Rehabilitation. doi: 10.1016/j.apmr.2016.03.023

Sirman, N., Beeke, S. \& Cruice, M. (2017) Professionals' perspectives on delivering conversation therapy in clinical practice. Aphasiology 31(4), 465-494.

http://www.tandfonline.com/doi/full/10.1080/02687038.2017.1278739 